\title{
Zur Verbindung einer transnationalen mit einer biographietheoretischen Perspektive in der Fluchtforschung: Migrationsverläufe im Kontext des syrischen Bürgerkrieges
}

Arne Worm

\section{Einleitung}

„Ach, auf dieser Route kommen Syrer nach Europa?" - Diese Frage begegnete mir in verschiedenen Varianten in privaten Gesprächen, aber auch in akademischen Zusammenhängen wiederkehrend, wenn ich von meiner Forschung zu Fluchtverläufen aus Syrien über den spanisch-marokkanischen Grenzraum um die Enklaven Ceuta und Melilla berichtete. Die Enklaven selbst waren den Fragenden in der Regel zwar ein Begriff. Ihre Grenzzäune sind geradezu als Ikonen einer „Festung Europa“ (vgl. Walters 2004: 692) in europäischen Mediendiskursen bekannt geworden. Der Befund, dass Geflüchtete aus dem syrischen Bürgerkrieg auf dieser geographisch nicht unbedingt naheliegenden Route nach Europa migrieren, sorgte hingegen eher für interessierte Verwunderung. Gegenüber der vor allem im Jahr 2015 wesentlich umfassender im Fokus stehenden „Balkanroute“ ${ }^{\text {"1 }}$ erschien die Flucht von Syrien über

${ }^{1}$ Im Jahresverlauf 2015 waren Hundertausende Geflüchtete aus Syrien, dem Irak, Afghanistan und anderen Ländern (Kasparek 2017: 102) vor allem von der Türkei über Griechenland in weitere EUStaaten eingewandert (vgl. Hess et al. 2017). Dies führte zu erheblichen politischen Diskussionen über

Worm, A. (2020): Zur Verbindung einer transnationalen mit einer biographietheoretischen Perspektive in der Fluchtforschung. Migrationsverläufe im Kontext des syrischen Bürgerkrieges. In: Babl, E./Becker, J. (eds.): Global Processes of Flight and Migration. The Explanatory Power of Case Studies / Globale Flucht- und Migrationsprozesse. Die Erklärungskraft von Fallstudien. Göttingen: Göttingen University Press, 103-122. bttps://doi.org/10.17875/ gup2020-1316 
Marokko bis nach Spanien wesentlich abwegiger bzw. eine Route des Umwegs. ${ }^{2}$ Auch ich selbst war zu Beginn meiner Feldforschung 2014 in den spanischen Enklaven überrascht, dass die größte Gruppierung in den lokalen Flüchtlingslagern Menschen aus Syrien waren. Je mehr ich auf Basis von vor allem familien- und lebensgeschichtlichen Interviews über ihre Fluchtverläufe aus dem gewaltsamen Konflikt in Syrien seit 2011 (vgl. hierzu Abboud 2016; Hinnebusch 2012) erfuhr, desto deutlicher wurden nicht nur die unterschiedlichen Fluchtkonstellationen. Vielmehr zeigte sich auch die Bedeutung grenzüberschreitender Netzwerke und Verflechtungen, innerhalb derer sich der Prozess, aus Syrien über Spanien/Marokko nach Europa zu migrieren, aufschichtete.

Dieser Befund verdeutlichte, dass meine und die in der einleitenden Frage ausgedrückte Verwunderung auf zwei sehr verkürzten Annahmen über Fluchtverläufe basierte: Erstens ging ich offenbar davon aus, dass diese relativ linear und unidirektional verliefen - von einem Herkunftskontext, den man aufgrund lebensbedrohlicher Bedingungen verlassen musste, in einen Fluchtkontext, in dem kurzfristig Schutz und längerfristig soziale Teilhabe gesucht wird. Demgegenüber zeigte sich in den untersuchten Fällen, wie erheblich diese Fluchtverläufe durch Etappen und Zwischenstationen geprägt waren. Zweitens zeigte sich, dass in die Frage nach der Route ein gewisses „Containerdenken“ (vgl. Beck 1997: 49 ff.) einging: Fluchtmigration erschien nicht nur als linearer Verlauf, sondern auch als Übergang zwischen distinkten, relativ abgeschlossenen und nationalstaatlich definierten sozialen Räumen. Mit anderen Worten: Der biographische Zugang konfrontierte mich zunehmend mit Dimensionen und Dynamiken von Flucht, die im Feld der Migrationsforschung unter dem Stichwort „Transnationalismus“ verhandelt werden. Die Fallauswertungen zeigten deutlich, wie bedeutsam - wenn auch unterschiedlich bedeutsam - transnationale Handlungsräume und Beziehungen für die Fluchtverläufe waren. Im Kontext der Flucht aus Syrien über Spanien/Marokko betraf dies vor allem Verflechtungen zwischen Syrien und Algerien. Für einige Geflüchtete waren zum Beispiel familial, berufsbiographisch oder politisch konstituierte Verflechtungen zwischen Syrien und Algerien bereits vor der Flucht regelmäßiger und selbstverständlicher Teil einer grenzüberschreitenden Lebensführung, während andere transnationale Netzwerke erst im Kontext der Flucht mobilisierten und wieder anderen diese Netzwerke weitestgehend fehlten. Interessanterweise erzeugten die Interviews und Gruppengespräche mit Geflüchteten aus Syrien selbst zunächst häufig den Eindruck eines relativ linearen Fluchtverlaufs , aus dem Krieg nach Europa“: Die Perspektiven und Selbstpräsentationen meiner Gesprächspartner*innen waren sehr stark auf die

die sogenannte „Flüchtlingskrise“ und in zahlreichen europäischen Staaten zum Ausbau restriktiver Migrations- und Grenzpolitiken.

${ }^{2}$ Gegenüber der „Balkanroute“ ist die Gesamtzahl der über Ceuta/Melilla nach Europa kommenden Menschen aus Syrien tatsächlich auch sehr gering. Laut Daten des UNHCR kamen in den Jahren 2014 2016 zwischen 2.500 und 8.000 Geflüchtete aus Syrien pro Jahr in die Enklaven. Online: https://data2.unhcr.org/en/documents/download/53859 und https://data2.unhcr.org/en/documents/download/46554 <03.02.2020>. 
prekären Lebenssituationen in den Flüchtlingslagern und - die eigene Flucht geradezu legitimierend - auf die Plausibilisierung der Kriegssituation in Syrien gerichtet (vgl. Worm 2019). Die Zwischenstationen und die komplexen Gesamtverläufe wurden - strukturell bedingt - verdeckt.

Ich möchte diese Ausführungen zum Ausgangspunkt nehmen, um die wesentlichen Anliegen dieses Artikels zu verdeutlichen: Welchen Beitrag leistet die Verbindung einer biographietheoretischen mit einer transnationalen Perspektive zur Untersuchung von Fluchtphänomenen? Inwiefern gelingt es mit dieser Verbindung, Flucht als komplexe Gesamtverläufe und eingebettet in sich wandelnde Beziehungsgeflechte bzw. Figurationen zu rekonstruieren?

Beginnen werde ich mit einigen Grundannahmen des biographietheoretischen Zugangs zu Fluchtphänomenen und einer transnationalen Forschungsperspektive (2). Daraufhin werde ich die Untersuchungs- und Projektkontexte sowie das methodische Vorgehen vorstellen, aus denen die empirische Basis dieses Beitrags hervorgeht (3). Darauffolgend diskutiere ich eine auf Basis „biographischer Fallrekonstruktionen“ (Rosenthal 1995) gebildete Typologie der unterschiedlichen Bedeutung transnationaler Verflechtungen für den Gesamtprozess von Fluchtverläufen (4). Der Beitrag endet mit konzeptionellen Überlegungen zu den Implikationen der Typologie und der Kombination einer transnationalen mit einer biographietheoretischen Perspektive für die Fluchtforschung ${ }^{3}(5)$.

\section{Grundzüge eines biographietheoretischen und transnationalen Zugangs zu Fluchtphänomenen}

Unter Fluchtmigrationen werden hier Formen der Migration verstanden, „deren Entstehungsbedingungen und deren Verläufe ... durch gewaltbedingte und gewalthervorbringende soziale Wandlungsprozesse und Ordnungsbildungen strukturiert sind“" (Worm 2019: 60). Mit dieser Konzeptualisierung kann die soziologische Untersuchung von Fluchtphänomenen einerseits aus einer statischen und binären Unterscheidung von Zwangs- und selbstgewählten Migrationen gelöst werden. Andererseits lenkt diese Definition den Blick auf das aus meiner Sicht wesentliche - und in seinen verschiedenen individuellen und kollektiven Folgen zu rekonstruierende Merkmal von Fluchtverläufen, nämlich durch kollektive Gewaltdynamiken verursacht und in ihrem Ablauf auf diese bezogen zu sein. Welchen Zugang bietet nun eine biographietheoretische Perspektive auf Flucht als Prozessgeschehen?

Grundsätzlich zielt ein soziologisch-biographietheoretischer Zugang in der sozialkonstruktivistischen Tradition (Schütz 1971[1953]; Berger/Luckmann 2007 [1969]) auf die Untersuchung sozialer Phänomene entlang der Rekonstruktion

\footnotetext{
${ }^{3}$ Die sich vor allem seit den 1980er Jahren institutionalisierende Flucht- und Flüchtlingsforschung hat sich in den letzten Jahren zu einem umfangreichen Forschungsfeld entwickelt. Für einen Überblick siehe Black 2001; Kleist 2015; Stepputat/Sørensen 2014. Ein Forschungsdesiderat hinsichtlich der Verbindung transnationaler Perspektiven mit der Fluchtforschung konstatieren Sauer et al. 2018.
} 
lebensgeschichtlicher Verläufe. Eine Biographie kann, so die Definition von Wolfram Fischer und Martin Kohli, als „sozialweltliches Orientierungsmuster" beziehungsweise als „soziales Konstrukt“ (1987: 26) der zeitlichen und prozesshaften Ordnung eines Lebens verstanden werden. Biographien entstehen und erhalten ihre Gestalt aus der wechselseitigen Beziehung zwischen Individuen und den soziohistorischen Kontexten, in die sie handelnd involviert sind (ebd.). Im Zentrum einer biographietheoretischen Perspektive steht folglich, diesen Prozess, das soziohistorische „Gewordensein“ eines lebensgeschichtlichen Verlaufs, zu rekonstruieren. Den Ansatzpunkt bilden hierbei die Erfahrungsbildung und die Handlungsweisen von Menschen in den verschiedenen Phasen ihres Lebens. Eine Lebensgeschichte erhält ihre Strukturierung im Prozess der Interpretation und Re-Interpretation von Erlebens- und Erfahrungszusammenhängen durch die handelnden Akteure in ihren jeweiligen gesellschaftlichen Kontexten (Rosenthal 1995). Artur Bogner und Gabriele Rosenthal (2017) schlagen mit der Verbindung eines biographietheoretischen Zugangs mit einer figurationssoziologischen Perspektive in Anschluss an die Arbeiten von Norbert Elias vor, diesen wechselseitigen Konstitutionsprozess von Individuen und Gesellschaft in längerfristige soziohistorische Wandlungsprozesse einzubetten sowie die Machtungleichheiten zwischen verflochtenen Individuen und Kollektiven zu berücksichtigen.

Migrationsphänomene sind aus einer biographietheoretischen und transnationalen Perspektive vielfach untersucht worden (vgl. z.B. Apitzsch 1999; Breckner 2005; Lutz 1991; Rosenthal 2004; Siouti 2013). Dabei beziehen sich einige Arbeiten direkt auf Fluchtmigrationen (Agha 1997; Inowlocki 2002; Rosenthal 1997; Rosenthal/ Bahl/Worm 2016). Als Perspektive auf Fluchtverläufe regt ein biographischer Zugang an, die Fluchtkonstellation und den Handlungsakt des Ausweichens vor drohenden oder bereits eingetretenen gefährdenden Lebenssituationen in den längerfristigen Horizont ihrer Entstehung und des weiteren Verlaufs (z.B. einzelne Stationen oder Lebensphasen der Flucht) einzubetten.

Diese Prozessperspektive beinhaltet, die aufrechterhaltenen, neu entstehenden und sich wandelnden sozialen Beziehungsgeflechte im Fluchtverlauf in die Analyse einzubeziehen, also jene Dimensionen, die unter dem Stichwort des „Transnationalismus" (Glick Schiller/Basch/Blanc-Szanton 1992) diskutiert wurden und werden. Unter diese Forschungsperspektive fallen zahlreiche Arbeiten, die sich mit der Bedeutung und den Prozessen der Aufrechterhaltung von Beziehungen von Migrierenden zu den Herkunftskontexten auseinandersetzen. Auch werden unter dieser Perspektive „transnationale soziale Räume“ (Pries 1996; Faist 2000) als grenzüberschreitende Verflechtungszusammenhänge, als Folge und Bedingung von Migrationsphänomenen untersucht. Auch wenn die Beziehungen von Migrierenden zu ihren Herkunftskontexten bereits in früheren Forschungsarbeiten berücksichtigt wurden, ${ }^{4}$ pointieren transnationale Perspektiven vor allem die Kritik am „,methodologi-

\footnotetext{
${ }_{4} \mathrm{Zu}$ nennen ist hier bereits die Untersuchung von William Isaac Thomas und Thomas Znaniecki zu polnischen Einwander*innen in Chicago zu Beginn des 20. Jahrhunderts (1958[1918-1920]).
} 
schen Nationalismus“ (Wimmer/Glick Schiller 2002): Indem die Verfasstheit und die Existenz von Nationalstaaten als zentrale Bezugskategorie der Forschung vorausgesetzt wurden, gerieten nicht nur soziale Verflechtungen jenseits der nationalstaatlichen Ebene aus dem Blick. Vielmehr sollten Nationalstaaten an sich nicht als einheitliche „Container“ (Beck 1997: 49 ff.) betrachtet werden. Der Blick ist vielmehr auf die komplexen Zwischenformen von Migrationen, Konstellationen des „Hier-und-Dort-Seins“, zu richten. Dies betrifft sowohl die Ebene von Zugehörigkeitsverortungen als auch die Ebene von Migrations- und Mobilitätspraktiken, mit denen soziale Räume hergestellt werden.

Bevor ich anhand der empirisch gebildeten Typologie verdeutliche, welche Dimensionen von Fluchtverläufen durch die Verbindung einer biographietheoretischen mit einer transnationalen Perspektive aufgezeigt werden können, führe ich kurz in die Untersuchungskontexte und Methoden ein, die die Typologie informiert haben.

\section{Untersuchungskontexte}

Die im folgenden Abschnitt dargestellte Typologie zur Bedeutung und zum Wandel transnationaler Verflechtungen im Fluchtkontext basiert auf (Teil-)Ergebnissen von zwei DFG-geförderten Forschungsprojekten. Das Projekt „Die soziale Konstruktion von Grenzgebieten. Ein Vergleich von zwei geopolitischen Fällen" ${ }^{5}$ untersuchte die Gegenwart und den soziohistorischen Wandel der marokkanisch-spanischen Grenzregion um die spanischen Enklaven Ceuta/Melilla im Vergleich mit der ägyptisch-israelischen Sinai-Grenze. Im Fokus des Projektes stand die Rekonstruktion der Erfahrungen, Handlungsweisen und Machtchancen der Mitglieder von Gruppierungen, die in den Grenzräumen aufeinander bezogen waren. Konkret heißt dies, dass wir uns mit den Handlungs- und Deutungsmustern der im Grenzraum ansässigen Lokalbevölkerungen, den staatlich-organisationalen Akteur*innen (zum Beispiel der Grenzpolizei; vgl. Bahl/Worm 2018; Ben-Ze’ev/Gazit 2018), Pendelarbeiter*innen (Bahl 2017) und (illegalisierten) Migrierenden verschiedener Herkunftskontexte (vgl. Rosenthal/Bahl/Worm 2016) beschäftigt haben.

Im Rahmen des Projektes habe ich selbst schwerpunktmäßig zu den Fluchtverläufen von Menschen gearbeitet, die aus dem syrischen Bürgerkrieg seit 2011 auf der Route über die spanischen Enklaven nach Europa migriert sind (vgl. Worm 2019). ${ }^{6}$

\footnotetext{
5 In diesem von Prof. Dr. Gabriele Rosenthal geleiteten vergleichenden DFG-Forschungsprojekt (RO 827/19-1; März 2014 - Februar 2019) forschten neben der Projektleiterin und dem Autor außerdem Eva Bahl sowie Prof. Dr. Efrat Ben-Ze'ev und Dr. Nir Gazit. Siehe auch online: https://www.unigoettingen.de/en/477891.html <18.02.2020>.

${ }^{6}$ Die an der nordafrikanischen Mittelmeerküste gelegenen Enklaven Ceuta und Melilla sind die einzigen Territorien eines europäischen Staates auf dem afrikanischen Kontinent mit einer Landgrenze zu einem afrikanischen Staat. Durch den Beitritt Spaniens zur „Europäischen Gemeinschaft" in den Jahren 1985/86 stellen die Grenzen dieser relativ kleinen Städte, jede hat gegenwärtig etwa 85.000 Einwohner*innen, eine EU-Außengrenze dar. Die seit Ende der 1990er-Jahre zunehmend erhöhten Grenzzäune um die beiden Städte standen wiederholt im Fokus der Berichterstattung internationaler Medien.
} 
Hierzu haben wir in den Enklaven neben Gruppengesprächen und kürzeren ethnographischen Interviews vor allem biographisch-narrative Gespräche mit Geflüchteten geführt. ${ }^{7}$ Orientiert am „Prinzip der Offenheit“ (Hoffmann-Riem 1980) sahen die verschiedenen Erhebungsformate jeweils vor, den Gesprächspartner*innen möglichst umfassend Raum zur Darstellung ihrer Perspektiven auf ihr vergangenes und gegenwärtiges Leben zu ermöglichen. Vor allem in den biographischen Interviews zeigte sich, dass die Darstellung der eigenen Lebens- und Familiengeschichte zunächst erheblich durch die gegenwärtige, überaus prekäre und unvorhersehbare Lebenssituation im Flüchtlingslager geprägt war. Die Gesprächspartner*innen konnten sich vielfach erst nach und nach auf eine Zuwendung zur Darstellung vergangener Lebensbereiche einlassen. Es war zentral, sich zunächst auf diese Rahmung einzulassen und zum Beispiel den erheblichen Frustrationen über die als blockiert wahrgenommene Lage in Ceuta oder Melilla oder das undurchsichtige Asylverfahren, aber auch den Ängsten und Sorgen bezüglich der Entwicklung des Konflikts in Syrien Raum zu geben (vgl. Worm 2019: 151). Erst im Verlauf der Gespräche und der Fallauswertung wurde der komplexe Gesamtverlauf der Flucht in seiner familien-, kollektiv- und lebensgeschichtlichen Einbettung deutlich. Für die Rekonstruktion der biographischen Genese der Fluchtkonstellation und der Fluchterfahrungen jenseits der prekären und unsicheren Lebenssituation „im Transit“ war es zudem äußerst gewinnbringend, mit Geflüchteten nach den Interviews in Melilla/Ceuta in Kontakt zu bleiben und zu einem späteren Zeitpunkt Nachgespräche zu führen. So erfuhren wir auch, wie einige Fluchtverläufe weitergingen und sich Prozesse der ReEtablierung und Teilhabe in Europa gestalteten. Hierbei traten vor allem die Unterschiede zwischen den Fluchtverläufen und die sozialen Entstehungsmechanismen dieser Unterschiede klarer hervor, die auch die folgende Typologie informiert haben.

Auf Teilhabeerfahrungen von Mitgliedern verschiedener Gruppierungen in Deutschland im Vergleich fokussiert auch das 2019 begonnene Projekt „Prozesse

\footnotetext{
Anders als Schwarze Geflüchtete, die im marokkanisch-spanischen Grenzraum erheblichen Repressionen ausgesetzt sind und für die der Eintritt in die Enklaven nahezu ausschließlich durch die lebensgefährliche Praxis der Überwindung der Grenzzäune möglich ist (vgl. Rosenthal/Bahl/Worm 2016), versuchen Geflüchtete aus Syrien - ebenfalls aufgrund fehlender legalisierter Zugänge -, in der Regel getarnt als „Marokkaner*innen“ im täglichen ,kleinen Grenzverkehr“ über die Grenze zu kommen. Auch diese Praxis ist überaus prekär und gefährlich. So droht bei Enttarnung durch die Grenzpolizei gewaltsame Zurückweisung, Familien werden in diesem Prozess getrennt, Gelder für falsche Pässe müssen aufgebracht werden (vgl. Worm 2019: 169 ff.).

${ }^{7}$ Als ethnografische Interviews bezeichne ich sämtliche Interviews und Gespräche, die sich im Rahmen der Forschung mit Akteur*innen im Feld ergeben haben und in denen nicht explizit zur Erzählung der Familien- und Lebensgeschichte aufgefordert wurde. Auch bei diesen Interviews haben wir uns im Projekt an den Prinzipien einer narrativen Gesprächsführung orientiert. Diese sieht vor, Interviewpartner*innen dabei zu unterstützen, ihre Perspektiven, Alltagserfahrungen und -handlungsweisen entlang eigener Relevanzen und textstrukturell in Form von Erzählungen darstellen (vgl. Schütze 1977; Rosenthal 2015: 163 ff.). Im Format des biographisch-narrativen Interviews wird den Interviewpartner*innen in einer ersten Phase mit einer offenen Erzählaufforderung Raum zur Darstellung der eigenen Familien- und Lebensgeschichte gegeben, bevor einzelne Lebensbereiche und Themen in den folgenden Interviewphasen vertieft werden.
} 
der Inklusion und Partizipation im Kontext sogenannter irregulärer Migration". 8 Ebenfalls aus einer biographietheoretischen Perspektive untersucht das Projekt Teilhabeerfahrungen und lebensgeschichtliche Verläufe von Migrierenden aus Syrien und Westafrika in Brasilien und in Deutschland. Ein Teil des Projektsamples setzt sich dabei aus Nachgesprächen mit Geflüchteten zusammen, die wir bereits zu einem früheren Zeitpunkt (z.B. im vorherigen Projekt) interviewen konnten. Die folgende Typologie transnationaler Verflechtungen basiert auf dem Vergleich von Fällen, mit denen im Rahmen dieses neuen Projekts Folgeinterviews in Anschluss an das Vorgängerprojekt geführt werden konnten, oder solchen, die im Rahmen des neuen Projektes erstmalig interviewt wurden. Die Fälle wurden mit dem Verfahren der „biographischen Fallrekonstruktion“ ausgewertet.

Bei biographischen Fallrekonstruktionen werden in getrennten Analyseschritten sowohl der lebensgeschichtliche Verlauf im Sinne der Erfahrungsaufschichtung im Prozess eines Lebens als auch dessen sprachliche Darstellung in Form einer erzählten Lebensgeschichte rekonstruiert (vgl. Rosenthal 1995). ${ }^{9}$ Das Verfahren sieht vor, die sequenzielle Gestalt des erfahrungs- bzw. lebensgeschichtlichen Verlaufs in seiner soziohistorischen Einbettung mit der sequenziellen Gestalt der gegenwärtigen Selbstpräsentation zu kontrastieren und die Differenz zu erklären. Hierdurch war es in den genannten Forschungskontexten z.B. möglich, aufzuzeigen, wie stark die Sicht der Interviewpartner*innen auf die gegenwärtigen Transitsituationen ihrer Flucht beschränkt war, warum die Flucht aus Syrien eher als linearer denn als brüchiger Prozess dargestellt wurde bzw. werden musste und wie durch die Vermeidung politisch heikler Selbstverortungen gegenüber anderen Geflüchteten bestimmte Lebensbereiche zunächst nur vorsichtig und rudimentär angesprochen werden konnten (vgl. Worm 2019: 257 ff.). Dieser Befund regte nämlich dazu an, den tendenziell verdeckten, aber erheblichen Differenzen in den Fluchtverläufen in den Gesprächen und Folgegesprächen nachzugehen sowie die Ursachen und Folgen dieser Differenzen bei der Rekonstruktion der familien- und lebensgeschichtlichen Verläufe herauszuarbeiten.

\section{Zur Bedeutung und zum Wandel transnationaler Verflechtungen im Fluchtverlauf: Eine Typologie}

Im vorherigen Abschnitt wurde in die Forschungskontexte eingeführt und der Befund erörtert, dass die komplexen Gesamtverläufe der Flucht - und mit ihnen die Bedeutung transnationaler Verflechtungen - innerhalb der auf die gegenwärtigen

\footnotetext{
8 In diesem ebenfalls von Prof. Dr. Gabriele Rosenthal geleiteten vergleichenden DFG-Forschungsprojekt (RO 827/21-1; Februar 2019 - Januar 2022) forschen neben der Projektleiterin und dem Autor außerdem Eva Bahl, Lucas Cé Sangalli, Dr. Sevil Çakır-Kılınçoğlu, Dr. Maria do Carmo dos Santos Gonçalves und Prof. Dr. Hermílio Santos. Siehe auch online: https://www.uni-goettingen.de/de/607273.html <19.02.2020>.

${ }^{9}$ Für eine komprimierte Darstellung des Verfahrens siehe Hinrichsen/Rosenthal/Worm 2013.
} 
Lebenssituationen fokussierten Selbstpräsentationen meiner Gesprächspartner*innen verdeckt wurden. Im Laufe der Forschung rückte aber nicht nur der Befund der generellen Bedeutsamkeit transnationaler Verflechtungen in den Fokus - dieser Befund ist in der Fluchtforschung nicht sonderlich neu (vgl. z.B. Shami 1996; Castles 2003). Vielmehr stellten sich die Fragen (a) nach der Bedeutung unterschiedlicher Qualitäten der transnationalen Netzwerke sowie (b) des Wandels der Netzwerke. Entlang dieser Dimensionen soll im Folgenden eine Typologie ${ }^{10}$ vorgestellt werden, die herausarbeitet, inwiefern der Einbindung in transnationale Handlungsräume strukturbildende Wirkung für Fluchtverläufe zukommt.

\subsection{Typus 1: Fluchtmigration innerhalb relativ stabiler transnationaler Handlungsräume}

Dieser Typus zeichnet sich dadurch aus, dass eine familien-, kollektiv- und lebensgeschichtlich aufgebaute Einbindung in transnationale Handlungsfelder im Kontext der Fluchtmigration weitestgehend stabil bleibt. Diese Einbindung ermöglicht zum einen, im Prozess des Ausweichens vor der existenziell bedrohlichen Kriegs- und Konfliktdynamik und der Re-Etablierung in einem anderen Gesellschaftskontext Ressourcen aus diesem Netzwerk zu mobilisieren (z.B. Informationen zu Routen sowie Lebensbedingungen an einem Fluchtziel; ökonomische Ressourcen; Unterkunft an Fluchtorten, etc.). Zum anderen bringt diese Einbindung mit sich, dass familien- und lebensgeschichtlich aufgebaute Orientierungs- und Handlungsmuster - wohlgemerkt: relativ - stabil bleiben, z.B. weil die Lebensführung vor der Flucht bereits mit der Teilhabe an Handlungsfeldern außerhalb des Herkunftskontextes verbunden war (durch berufliche Tätigkeit oder Familiennetzwerke), an die im Fluchtverlauf tendenziell angeschlossen werden kann. Die relative Stabilität dieser transnationalen Handlungsfelder ist erheblich von der konkreten Kriegsdynamik sowie Öffnungs- oder Schließungsprozessen innerhalb von Grenz- und Migrationsregimen abhängig.

Die untersuchten Fälle deuten darauf hin, das Repräsentant*innen dieses Typus aus eher urbanen und sozioökonomisch etablierten Familienmilieus kommen und im Rahmen der Flucht vor der Gewalt in Syrien auf - relativ betrachtet - privilegierte Migrationsformen zurückgreifen können (z.B. indem ein „regulärer“ Studienaufenthalt in Deutschland organisiert wird). Damit soll keineswegs bestritten werden, dass Vertreter*innen dieses Typus erheblich unter der massiven Gewalteskalation in Syrien gelitten haben und von ihr auch im Fluchtverlauf weiter betroffen sind. Die Fälle deuten vielmehr an, dass Vertreter*innen dieses Typus sich durch die bestehen-

\footnotetext{
${ }^{10}$ Hierbei orientiere ich mich an der Typenbildung als Variante theoretischer Verallgemeinerung, wie sie von Rosenthal (2015: 79 ff.) im Anschluss an Max Weber und Kurt Lewin diskutiert wird. Ein Typus ist demnach weder eine rein abstrakte, von konkreten soziohistorischen Konstellationen abgehobene Modellbildung noch besteht der Typus lediglich aus der beschreibenden Nachzeichnung sämtlicher Fallbestandteile. Vielmehr sind anhand von Fallrekonstruktionen gebildete Typen erklärende Modelle zu den Konstitutionsregeln eines Fallgeschehens (ebd.).
} 
de Verbindung zur (Groß-)Familie und zu Freunden im Herkunftskontext erheblich an der Diskrepanz zwischen deren sehr bedrohlichen Lebenssituationen und der eigenen Situation ,relativer" Sicherheit abarbeiten. Insofern basiert die relative Stabilität dieses Typus auf Formen der biographischen „Orientierungsarbeit“ (FischerRosenthal 1995: 44) der Geflüchteten, also der - im Kontext von Flucht schwierigen - sinnhaften Kontinuitätsherstellung zwischen dem Leben vor und im Prozess der Flucht sowie die Aufrechterhaltung von auf den Herkunftskontext bezogenen Handlungsroutinen (z.B. durch politisches Engagement).

Dieser Typus wird z.B. repräsentiert durch den Fall von Sufian Khalifa, ${ }^{11}$ dessen Fluchtverlauf im familien- und lebensgeschichtlichen Kontext im Folgenden skizziert wird. Sufian, geboren Mitte/Ende der 1990er Jahre, flüchtete im Jahr 2015 aus seiner Herkunftsstadt Damaskus über die Türkei, Griechenland und die „Balkanroute" bis nach Deutschland. Zum Zeitpunkt des biographischen Interviews Ende des Jahres 2019 studierte Sufian seit drei Semestern Physik in einer nordrhein-westfälischen Universitätsstadt. Wie bei anderen von uns interviewten jungen Männern aus Syrien, bildete der drohende Einzug zum Militärdienst den unmittelbaren Fluchtanlass. Innerhalb der fragmentierten Bürgerkriegssituation (vgl. Abboud 2016: 84 ff.) und der drohenden Involvierung in die lebensbedrohlichen Kämpfe zwischen dem syrischen Militär und bewaffneten Oppositionsgruppen entschied sich Sufian, Syrien zu verlassen, nachdem das Alltagsleben für die Familie in Damaskus in den Jahren zuvor wiederkehrend erheblich durch Bürgerkriegsgewalt geprägt gewesen war. Diese Konstellation verband sich mit der familialen Aspiration, Sufian solle auBerhalb Syriens seine Bildungskarriere durch ein Studium fortsetzen. Sufian selbst konzentrierte sich im ersten, von ihm selbst gestalteten Teil des Interviews ganz auf diese Grobstruktur der Flucht. Wie erheblich die verschiedenen Komponenten der Fluchtkonstellation und der weitere Verlauf mit familien- und lebensgeschichtlich konstituierten Dynamiken verwoben waren und inwiefern darin eine transnationale Orientierung relevant wurde, zeigte sich erst durch Vertiefungsfragen im Interview und schließlich durch die Einbettung des Falles in den soziohistorischen Kontext im Rahmen der fallrekonstruktiven Auswertung.

Aus dem Interview und Kontextrecherchen ließ sich rekonstruieren, dass die Familie einem konservativen sunnitischen Mittelschichtsmilieu in Damaskus angehört. Beide Eltern verfügen über Hochschulabschlüsse, die sie im Zuge einer autoritären Modernisierung ,von oben“ (vgl. Hinnebusch 2001, 2012), z.B. der Ausweitung des Staats- und Bildungssektors in Syrien seit den 1950er/1960er Jahren, realisieren konnten. Der Vater ging einem technischen Beruf nach; die Mutter war Lehrerin. Darüber konnte die Familie zwar zum einen eine sozioökonomisch relativ gesicherte Positionierung erlangen. Gleichzeitig standen gerade Mitglieder des urbanen, sunnitisch-konservativen und mittleren Milieus aber auch in Distanz zum (alawitischen) Regime (vgl. Perthes 1990: 90 ff.). Bildungsaspiration bei gleichzeitiger

${ }^{11}$ Alle Namen von Interviewten und Falldetails, die einen Rückschluss auf die Person zulassen, wurden verändert. Das biographische Interview mit Sufian wurde im Dezember 2019 in Deutschland von mir zusammen mit Tom Weiß in deutscher Sprache geführt. 
Distanzierung zum Regime stellte entsprechend das wesentliche familiale Sozialisationsumfeld Sufians dar. Erst sehr spät im Interview und vorsichtig thematisierte er, dass sein Vater in den frühen 1980er Jahren als Mitglied einer sunnitischen Oppositionsgruppe im Gefängnis war. Die Haftzeit, in der der Vater vermutlich Folter erleiden musste, steht im Kontext einer Phase erheblicher politischer Auseinandersetzungen zwischen dem syrischen Regime und oppositionellen sunnitischen Gruppen. Diese Auseinandersetzungen kulminierten im Februar 1982 im „Massaker von Hama“, bei dem nach unterschiedlichen Schätzungen zwischen 10.000 und 40.000 Menschen durch das syrische Militär und regimenahe Milizen getötet wurden (Lefèvre 2013: 128). Korrespondierend mit dem offiziellen Diskurs, in dem das Massaker bis heute geleugnet und tabuiert wird, wurde über diese Lebensphase auch in Sufians Familie nicht offen gesprochen. Dieses Tabu beinhaltete auch die Flucht eines vermutlich ebenfalls in der Gruppe aktiven Onkels (väterlicherseits) nach Deutschland in dieser Phase. Im Interview beginnt Sufian selbst, darüber zu reflektieren, wie ihm in verschiedenen Situationen ein positives Bild von Deutschland vermittelt wurde, ohne dass er damals darüber nachgedacht habe, in welchem Kontext sein Onkel nach Deutschland migriert war. Aus der Verbindung der politischen Vergangenheit des Vaters und der politischen Distanz zum Regime bei gleichzeitig bestehenden Bildungsanforderungen an Sufian ergab sich nicht nur, dass er einen groBen Teil seiner Schulzeit in privaten Bildungseinrichtungen verbrachte. Vielmehr zeigte sich auch, dass von den Eltern vor allem eingefordert wurde - beispielsweise indem die Kinder Englisch lernen sollten -, sich auf die Option vorzubereiten, Syrien zu verlassen. Im erweiterten Familienumfeld studierte z.B. bereits vor Beginn des Konflikts in Syrien im Jahr 2011 ein Cousin in Istanbul. Weitere Freunde flohen ebenfalls dorthin. Als sich die Gewalteskalation in Syrien im Fall von Sufian zu der Konstellation aufschichtete, Syrien verlassen zu müssen, griff er zunächst auf dieses transnationale Familien- und Freundesnetzwerk zurück. Kurzfristig erlebte er - so beschrieb er es im Interview - das Leben in Istanbul als „nicht viel anders“ zum Leben in Damaskus vor dem Konflikt.

Im Austausch mit seinem in Deutschland lebenden Onkel und dessen Familie die ihm u.a. anboten, ihm das Geld für die Fluchtroute zu leihen, entschied sich Sufian, aus der Türkei nach Deutschland zu migrieren. Es fällt ihm im Interview schwer, die mit erheblichen Gefahren verbundene Fahrt mit dem Boot nach Griechenland zu thematisieren. Auch die weitere Migration nach Deutschland auf der „Balkanroute“, die er innerhalb einer Woche bewältigte, beschreibt er im Interview relativierend als „the normal route“ und „I got lucky“. In Deutschland erhielt Sufian Mitte 2016 einen Flüchtlingsstatus - begünstigt durch eine asylpolitische Konstellation, in der dieser Status Geflüchteten aus Syrien häufig recht zügig zuerkannt wurde. Mit Hilfe der bereits seit vielen Jahren in Deutschland lebenden Familienmitglieder konnte Sufian seine Lebenssituation relativ schnell stabilisieren. Er selbst hebt den erheblichen Vorteil von Englischkenntnissen hervor.

Mit der - auch familial erwarteten - Fortsetzung eines Studiums repräsentiert der Fall von Sufian einen Fluchtverlauf, bei dem sowohl die Verfügbarkeit transna- 
tionaler Netzwerke als auch eine im Familienumfeld nahegelegte transnationale Orientierung nicht nur konstitutiv, sondern ausgesprochen stabilisierend waren. ${ }^{12}$

\subsection{Typus 2: Fluchtmigration innerhalb labiler und sich wandelnder transnationaler Handlungsfelder}

Der zweite Typus repräsentiert Fluchtverläufe, die zwar einerseits durch transnationale Netzwerke und Handlungsweisen strukturiert sind, die sich aber andererseits in diesem Prozess erheblich wandeln. Dies umfasst sowohl Fälle, die eher lose Netzwerke im Prozess der Flucht kurzfristig mobilisieren können, ohne dass diese Einbindung notwendigerweise zur längerfristigen sozialen Teilhabe im Fluchtkontext beiträgt. Es umfasst zum anderen Fälle, in denen relativ stabile transnationale Handlungsweisen im Kontext der Flucht brüchig werden oder zusammenbrechen. Dieser Wandel hängt mit der Kriegs- und Konfliktdynamik im Herkunftskontext, dem Wandel von Migrations- und Grenzregimen zwischen Herkunfts- und Ankunftskontexten sowie mit einer prekärer werdenden Lebenssituation im Ankunftskontext zusammen. Die im Folgenden erörterten Fallbeispiele verbindet, dass sich die transnationale Einbindung des eigenen Lebens im Fluchtverlauf erheblich wandelte.

Während der Forschungsaufenthalte in Melilla und Ceuta zwischen 2014 und 2017 wurde deutlich, dass ein Teil der Geflüchteten aus Syrien bereits in den Jahren vor der Flucht kürzere oder längere Lebensphasen außerhalb Syriens verbracht hat, mehrfach in andere - vor allem arabische - Staaten gependelt ist, und/oder in transnationalen Familienkonstellationen gelebt hat. Durch den Kriegsverlauf - aber auch durch grenzpolitische Schließungsprozesse in Reaktion auf Fluchtbewegungen wurde diese transnational orientierte Lebensführung zunehmend unmöglich. Wie ich an den folgenden Fallbeispielen verdeutlichen werde, betraf dies in Bezug auf die Fälle, deren weiterer Fluchtverlauf über Spanien/Marokko führte, vor allem Verflechtungen zwischen Algerien und Syrien.

Eine Variante dieses Typus repräsentiert z.B. der Fall von Maher Saifi (geboren ca. 1970) und seiner Familie. ${ }^{13}$ Maher, der aus einer syrisch-palästinensischen Familie aus Damaskus stammt, lebte bereits einige Jahren in Algerien, als seine Ehefrau, zwei Söhne und zwei Töchter im Jahresverlauf 2014/2015 dorthin flüchteten. In dieser Phase hatten bewaffnete Gefechte im Damaszener Viertel Jarmuk - einem ehemaligen palästinensischen Flüchtlingslager - erheblich zugenommen und es hatte sich die dortige Versorgungslage dramatisch verschlechtert. Im Familienkontext stellte ihre Flucht aus der lebensbedrohlichen Lage in Jarmuk bereits die dritte Erfahrung

\footnotetext{
${ }^{12}$ In der bisherigen Projektstichprobe der Forschung in Deutschland fallen etliche Fälle - im Übrigen nicht nur aus Syrien - in diesen Typus. Dies lässt sich relativ einfach dadurch erklären, dass die Fluchtmigration nach Deutschland für Vertreter*innen dieses Typus alltagsweltlich naheliegender und zu bewerkstelligen ist als für Vertreter*innen der anderen Typen.

${ }_{13}$ Mit Maher Saifi führten Ahmed Albaba und ich im September 2014 zwei biographisch-narrative Interviews in Melilla. Die Interviews wurden auf Arabisch mit deutscher Übersetzung geführt. Im Frühjahr 2020 kontaktierten wir Maher, inzwischen mit seiner Familie in Deutschland lebend, für ein telefonisches Nachgespräch.
} 
erzwungener Migration dar: Mahers Großeltern und Eltern (als Jugendliche) waren wie viele Tausende Palästinenser*innen im Verlauf des Arabisch-Israelischen Krieges von 1948/1949 nach Syrien geflüchtet. ${ }^{14}$ Maher selbst flüchtete Anfang der 1990er Jahre nach Algerien, nachdem er viele Jahre im Libanon für eine bewaffnete palästinensische Oppositionsgruppe aktiv war - eine Phase, in der sein Leben zwischen dem partiell durch syrisches Militär besetzten Libanon und der Familie in Jarmuk pendelte. Maher sollte wegen seiner Mitgliedschaft in einer verbotenen palästinensischen Partei in Syrien verhaftet werden. Bei der Flucht nach Algerien halfen ihm Netzwerkkontakte innerhalb der Partei. ${ }^{15}$

Mahers Leben war bis zur Fluchtmigration nach Europa dadurch geprägt, dass er in Algerien begann, für ein internationales Bauunternehmen zu arbeiten, für das er beruflich überwiegend in verschiedenen arabischen Ländern, aber auch in Südeuropa tätig war. Der Familie in Jarmuk schickte er einen Teil seines Einkommens. Als dieses transnationale Familienmodell durch den Kriegsverlauf in Syrien brüchig wurde, flüchtete Mahers Kernfamilie zu ihm nach Algerien. Die Möglichkeit, nach Algerien zu fliegen, setzte natürlich das ökonomische Kapital für die Flugtickets voraus. Ansonsten konnten Menschen aus Syrien in dieser Phase des Konflikts noch relativ einfach nach Algerien einreisen. Mit der Zunahme von Fluchtmigrationen aus Syrien nach Algerien wurden die Visaregelungen für syrische Staatsbürger*innen (bzw. auch Palästinenser*innen aus Syrien) im Frühjahr 2015 verschärft. Wie bei anderen untersuchten Fällen war sein Migrationsprojekt zu diesem Zeitpunkt nicht oder nicht in erster Linie darauf ausgelegt, Algerien als Transitroute nach Europa zu nutzen (vgl. Worm 2019: 226). Ebenfalls wie in anderen Fällen waren Maher und seine Familie bei der Re-Stabilisierung ihres Lebens in Algerien vor allem auf sich selbst - bzw. informelle Netzwerke - angewiesen. Maher thematisierte im Interview in Melilla - wie andere Geflüchtete aus Syrien auch -, dass die Diskurse über die neuankommenden syrischen Geflüchteten in Algerien zunehmend stigmatisierend und problematisierend wurden. Maher sah vor allem für seine Kinder im jungen Erwachsenenalter keine langfristige Lebensperspektive in Algerien, während eine Rückkehr nach Syrien aufgrund des anhaltenden Konfliktverlaufs auf unbestimmte Zeit unmöglich war. Im Kontext seiner eigenen Tätigkeit in verschiedenen Ländern erschien ihm die Fortsetzung der Fluchtmigration nach Europa naheliegend, wobei die lebensgefährliche Flucht über das Mittelmeer vermieden werden sollte. Folglich entschied sich die Familie für den Weg über Spanien/Marokko, obwohl auch dieser Weg prekär und gefährlich ist: Zur Überquerung der Landgrenze zwischen Algerien und Marokko, die wegen des Konflikts um die Westsahara seit 1994 offiziell geschlossen ist, waren Maher und seine Familie auf die illegalisierte Beförderung durch „Schleuser“ angewiesen.

14 Von den insgesamt etwa 700.000 in dieser Zeit in Nachbarländer geflüchteten und vertriebenen Palästinenser*innen gingen etwa 90.000 bis 100.000 nach Syrien (Brand 1988: 621).

15 Dies steht im Kontext der Unterstützung der „Palästinensischen Befreiungsorganisation“ (PLO) durch den algerischen Staat (vgl. Heller 1995: 82 ff.). 
Im Fall von Maher (und seiner Familie) zerbricht ein transnationales Familienmodell im Zuge des Krieges und kann nur kurzzeitig für die Flucht mobilisiert werden.

Ähnlich zeigte sich dies im Fall von Jamil Farajeh und seiner Familie. ${ }^{16}$ Auch Jamils Familie kann bei ihrer Flucht vor der kollektiven Gewalt in ihrem Damaszener Viertel auf das Familiennetzwerk zu einer in Algerien lebenden Tante zurückgreifen. Auch bei Jamil, dessen Vater - wie Maher - einen palästinensischen Hintergrund hat, konstituierte sich das „Fluchtziel““ Algerien neben der konkreten Verbindung zur Familie der Tante durch das positive Fremdbild über Algerien als „palästinenserfreundliches" Land.

Einige Zeit nach ihrer Flucht nach Algerien reiste Jamils Mutter wiederholt kurzzeitig zurück nach Damaskus, um Jamils Großmutter zu versorgen und sie später nach Algerien zu holen. Dies ist ein bemerkenswertes Beispiel für die „NichtLinearität" der Fluchtverläufe und vor allem die prekäre Aufrechterhaltung transnationaler Räume auch unter massiven Konfliktbedingungen. Für Jamils Familie war die Rückkehr von einzelnen Familienmitgliedern nach Syrien jedoch mit der erheblichen Angst verbunden, dass diese nicht wieder zurückkehren könnten. Mit der Verschärfung der Visaregelungen für Syrer*innen in Algerien im Frühjahr 2015 endete diese Mobilität, während der Krieg in Syrien eine Rückkehr für unbestimmte Zeit unmöglich machte. Diese Faktoren trugen - zusammen mit einer sehr prekären Lebenslage und der fehlenden langfristigen Perspektive in Algerien - dazu bei, die Fluchtmigration nach Europa fortzusetzen.

Zusammenfassend zeichnet sich dieser Typus dadurch aus, dass transnationale Verflechtungen im Fluchtverlauf zwar partiell aufrechterhalten oder mobilisiert werden können, aber im Fluchtverlauf labil bleiben oder gar zusammenbrechen. In diesem Fall „schichtet“ sich die Entstehung und Fortsetzung der Fluchtmigration gerade durch die Wandlungsdynamik innerhalb dieses labilen Netzwerkes auf.

\subsection{Typus 3: Fluchtmigration ohne Einbindung in transnationale Handlungsfelder}

Die Strukturmerkmale, die den dritten Typus konstituieren, lassen sich recht einfach aufzeigen: Die Flucht vor der Kriegsdynamik in einen anderen Gesellschaftskontext findet weitestgehend ohne Einbindung in transnationale Handlungsfelder statt - sowohl auf der Ebene der biographischen Orientierung als auch im Sinne von Ressourcen aus diesem Netzwerk. Vertreter*innen dieses Typus, die sich mit der Flucht vor Gewalt über die syrischen Landesgrenzen hinweg vor existentieller Bedrohung in Sicherheit bringen, verfügen in den Transit- oder Ankunftskontexten nicht über konkrete soziale Netzwerke, die z.B. über familien- oder kollektivgeschichtlich aufgebaute Zugehörigkeiten mobilisiert werden können. Die Vertreter*innen dieses Typus sind dadurch in den jeweiligen Fluchtkontexten besonders benachteiligt und

${ }^{16}$ Für eine ausführliche Fallrekonstruktion zu diesem Fall siehe Worm 2019: 175 ff. 
verwundbar. Dass Mobilität - gerade auch im Kontext von Fluchtmigration - eine erhebliche soziale Ungleichheitsdimension umfasst, zeigt sich gerade im Kontrast der Vertreter*innen dieses Typus mit den beiden Typen zuvor. Sie sind zudem durch Schließungsprozesse innerhalb von Migrationsräumen besonders benachteiligt. Beides lässt sich exemplarisch am Fluchtverlauf von Rima und Samir al-Omar verdeutlichen. ${ }^{17}$

Der Fluchtverlauf von Rima und Samir al-Omar steht im kollektiv- und lebensgeschichtlichen Kontext einer erheblichen Marginalisierung als syrische Turkmen*innen, der Desertation von Samir zunächst aus dem syrischen Militär und später aus der oppositionellen Al-Nusra-Front sowie massiver Gewalt durch bewaffnete Kämpfe in ihrer Herkunftsstadt Idlib. Samir wurde, nachdem er im Konfliktverlauf als Soldat aus dem syrischen Militär geflüchtet war, verhaftet und schwer gefoltert. Auch für Rima, die weiterhin im schwer umkämpften Idlib lebte, war diese Phase eine erhebliche Leidenszeit. Als sie sich entschieden, Syrien zu verlassen, war mit der Flucht vor der unmittelbaren Gewalt in Idlib verbunden, in Syrien längerfristig keine Lebensperspektive mehr zu haben. Dem Ziel und der Notwendigkeit, sich in einem anderen Land - möglichst weit entfernt vom syrischen Kontext - ein neues Leben aufzubauen, standen das Fehlen transnationaler Netzwerke sowie neueingeführte Grenzschließungen auf verschiedenen Fluchtrouten (z.B. zwischen der Türkei und Griechenland; nach Algerien) gegenüber. Zudem war es aus Samirs Positionierung als Deserteur heraus unmöglich, Syrien mit dem Flugzeug zu verlassen. Samir und Rima flüchteten in den Libanon, dessen Grenzen zu Syrien auch in dieser Konfliktphase relativ einfach zu überqueren waren. Von dort flüchteten Samir und Rima in die Türkei, von wo aus sie nach Mauretanien flogen. Mauretanien war in dieser Phase eines der wenigen Länder, in das Syrer*innen zur Einreise kein Visum benötigten. ${ }^{18}$ Bei der anschließenden lebensgefährlichen Route durch Mali und über die formal geschlossene Grenze nach Algerien waren Samir und Rima auf „Schmuggler“" angewiesen. Ihre Fluchtroute nach Europa gestaltete sich somit durch das Fehlen transnationaler Netzwerke wesentlich prekärer als in den anderen skizzierten Fällen.

\section{$5 \quad$ Zusammenfassung}

In diesem Beitrag wurde die Bedeutung einer transnationalen in Verbindung mit einer biographietheoretischen Perspektive für die Untersuchung von Fluchtphänomenen erörtert. Die vorgestellte Typologie, als ein Ergebnis dieser Verbindung, verweist auf Sachverhalte, deren methodische und theoretische Konsequenzen für die

\footnotetext{
17 Rima und Samir wurden im März 2017 von Gabriele Rosenthal, Dolly Abdul Karim und mir auf Arabisch mit deutscher Übersetzung in Melilla interviewt. Für eine ausführliche Fallrekonstruktion zu diesem Fall vgl. Worm (2019: 227 ff.). Leider haben wir den Kontakt zu Rima und Samir nicht wiederherstellen können.

${ }^{18}$ In Mauretanien wurde im Verlauf des Jahres 2016 eine Visumspflicht für syrische Staatsbürger*innen einführt. Online: https://www.unhcr.org/news/stories/2017/3/58b94b914/struggling-syrians-mauritania-finland-offers-fresh-hope.html $<28.04 .2020>$.
} 
Flucht- und Flüchtlingsforschung bedeutsam sind: Fluchtverläufe sind auf unterschiedliche Weisen auf transnationale Verflechtungen bezogen. Diese bedingen sowohl den geographischen Verlauf der Flucht als auch - was ich für soziologisch folgenreicher und interessanter halte - die Handlungsmöglichkeiten und das Erleben der Fluchtverläufe. Die Einbindung in transnationale Verflechtungen spiegelt hierbei Unterschiede in den lebens-, familien- und kollektivgeschichtlichen Verläufen vor der Flucht und damit ungleiche Machtbalancen zwischen Geflüchteten wider.

Die Befunde, die hier am Beispiel von Fluchtverläufen aus Syrien expliziert wurden, stellen meine Kolleg*innen und ich im aktuellen Forschungsprojekt in strukturell gleicher Weise auch im Falle der Geflüchteten aus westafrikanischen Kontexten fest. Auch hier unterscheidet sich die Verfügbarkeit transnationaler Netzwerke und Ressourcen je nach familien- und kollektivgeschichtlichem Kontext erheblich. Für die Fluchtforschung ist allerdings nicht nur der Befund der Bedeutsamkeit transnationaler Verflechtungen an sich relevant, sondern insbesondere der Verlaufscharakter des Wandels. Konkret bedeutet dies zu fragen, wie transnationale Verflechtungen durch Migrationen und Mobilität unter Gewalt-, Kriegs- und Konfliktdynamiken entstehen, aufrechterhalten werden und sich verändern. Es bedeutet zudem, den Fragen nachzugehen, welche divergierenden Folgen für Individuen und soziale Kollektive dieser Wandel mit sich bringt. Auch Veränderungen innerhalb der Migrationsräume sowie der Transit- und Ankunftskontexte spielen hier eine erhebliche Rolle (vgl. Tonah in diesem Band am Beispiel des Migrationsraums WestafrikaLybien).

In den empirischen Fallbeispielen zu den Fluchtverläufen aus Syrien wird deutlich, wie erheblich sich die Migrations- und Teilhabeoptionen innerhalb und zwischen verschiedenen Gruppierungen von Geflüchteten unterscheiden: Soziohistorisch längerfristig entstandene transnationale Verflechtungen waren für die Mitglieder mancher Gruppierungen und Milieus bereits vor der Flucht selbstverständlicher Teil transnationaler Handlungspraktiken (z.B. ökonomisch, familial oder politisch). Manche konnten latente Netzwerke während der Migrationen reaktivieren, während anderen Geflüchteten diese Netzwerke weitestgehend fehlten. Zudem zeigte sich in den Projektzusammenhängen auch deutlich, wie Prozesse der Teilhabe von Geflüchteten nicht nur auf divergierende Bedingungen in den verschiedenen Transitund Ankunftskontexten (z.B. Deutschland, Algerien) abgestimmt werden müssen. Sie müssen vielmehr auch mit der Aufrechterhaltung transnationaler, auf die Kriegsund Konfliktdynamik bezogener Handlungspraktiken koordiniert werden (z.B. die Aufrechterhaltung der Involvierung in zivilgesellschaftlichen Oppositionsbewegungen in Syrien; Unterstützung von Familienmitgliedern, etc.). Hinzu kommt der Befund, dass Fluchtphänomene sich nicht auf den singulären Akt des Ausweichens vor Gewalt, Krieg und Verfolgung beschränken lassen, sondern in Etappen verlaufen, potentiell sogar eine kurzzeitige Rückkehr in den lebensbedrohlichen Herkunftskontext beinhalten können. Die Fallbeispiele verweisen insbesondere darauf, dass transnationale Netzwerke vor allem auf der Ebene familialer Verflechtungen hergestellt werden und dadurch wirkmächtig und handlungsrelevant werden. Sie zeigen zudem 
die für die Flucht über Spanien/Marokko konkrete Bedeutung von Verflechtungen zwischen Syrien und Algerien.

Soziologisch hilft diese weiter zu differenzierende Typologie als „sensibilisierendes Konzept" (Blumer 1954) innerhalb der Fluchtforschung, diesen Unterschieden genauer nachzugehen. Zum Beispiel könnte diese Perspektive den vielfach herausgestellten und doch gegenüber hiesigen Diskursen nach wie vor erwähnenswerten Befund erörtern helfen, dass sich Fluchtmigrationen weitestgehend in und zwischen Gesellschaften des ,globalen Südens“ abspielen und nur eine relativ geringe Zahl von Menschen in Staaten der Europäischen Union flüchtet. Hier kann eine transnationale und lebensgeschichtliche Perspektive dazu beitragen, genauer zu untersuchen, wie diese Netzwerke entstehen und sich wandeln.

Eine biographietheoretische und eine transnationale Perspektive informieren sich insofern wechselseitig, als sie den Fokus auf den Gesamtprozess der Entstehung, Aufrechterhaltung und des Zerfalls transnationaler Bindungen und Handlungsformen analytisch greifbar machen. Anstelle einer vereinfachenden, mechanistischen Perspektive auf den Zusammenhang von „Krieg“ und „Flucht“ kann ein biographieanalytischer Zugang empirisch herausarbeiten, wie sich das Erleben der Konfliktdynamik und die Genese von „Fluchtentscheidungen“ und -verläufen innerhalb von lebens-, familien- und kollektivgeschichtlichen Prozessen herausgebildet beziehungsweise aufgeschichtet haben. Auch werden Fluchtmigrationen mit diesem Zugang nicht auf eine „singuläre“ Handlung der Flucht oder auf einzelne „Stationen" oder Phasen im Fluchtverlauf (zum Beispiel das Leben im Krieg; Grenzarrangements; das Leben und die administrativen Praktiken im Flüchtlingslager) reduziert. Vielmehr werden der gesamte Verlauf der Fluchtmigration und die ihn hervorbringenden sozialen Dynamiken, Verflechtungen sowie die Handlungs- und Deutungsweisen der Geflüchteten in den Blick genommen.

Die Zwischenbefunde des laufenden Forschungsprojektes, die in die diskutierte Typologie eingegangen sind, deuten darauf hin, dass die Teilhabeerfahrungen von Geflüchteten - nicht nur aus Syrien - in Deutschland wie auch in Brasilien erheblich von den transnationalen Verflechtungen, in denen die Geflüchteten stehen, abhängen. Ich möchte diesen Beitrag mit einer These beschließen, die sich aus einem Vergleich zwischen den hier thematisierten Befunden zu den Fluchtverläufen aus Syrien mit weiteren empirischen (Zwischen-)Befunden des aktuellen Forschungsprojektes (z.B. zur Bedeutung religiöser Netzwerke von Migrierenden aus dem Senegal in Brasilien) anbietet: Die unterschiedliche Einbindung von Geflüchteten in transnationale Netzwerke, die Unterschiede hinsichtlich der Stabilität und des Wandels dieser Netzwerke und die ungleiche Verteilung kollektiv- und familiengeschichtlich aufgebauter transnationaler Orientierungen und Ressourcen stellt eine relevantere Komponente für Teilhabeerfahrungen und -chancen in den „Ankunftskontexten“ dar als der (nationalstaatliche) Herkunftskontext an sich. 


\section{Literatur}

Abboud, S. (2016): Syria. Cambridge: Polity.

Agha, T. (1997): Lebensentwürfe im Exil. Biographische Verarbeitung der Fluchtmigration iranischer Frauen in Deutschland. Frankfurt a. M./New York: Campus.

Apitzsch, U. (Hrsg.) (1999): Migration und Traditionsbildung. Opladen: Westdeutscher Verlag.

Bahl, E. (2017): Precarious Transnational Biographies. Moroccan Juveniles in the Spanish Enclaves of Ceuta and Melilla. In: Rosenthal, G./Bogner, A. (Hrsg.): Biographies in the Global South. Life Stories Embedded in Figurations and Discourses. Frankfurt a. M./New York: Campus, 185-208.

Bahl, E./Worm, A. (2018): Biographische und ethnographische Zugänge zu WirBildern, Sie-Bildern und Handlungspraktiken in einer Organisation. Die spanische Polizeieinheit Guardia Civil in Ceuta und Melilla. In: Zeitschrift für Qualitative Forschung, 19(1/2), 233-251.

Beck, U. (1997): Was ist Globalisierung? Irrtümer des Globalismus. Antworten auf Globalisierung. Frankfurt a. M.: Suhrkamp.

Ben-Ze'ev, E./Gazit, N. (2018): Juggling Logics on the Egyptian-Israeli Borderland. Soldiers Between Securitization and Arbitrary Humanitarianism. In: Journal of Contemporary Ethnography, 47(2), 255-277.

Berger, P. L./Luckmann, T. (2007[1969]): Die gesellschaftliche Konstruktion der Wirklichkeit. Eine Theorie der Wissenssoziologie. Frankfurt a. M.: Fischer.

Black, R. (2001): Fifty Years of Refugee Studies. From Theory to Policy. In: International Migration Review, 35(1), 55-78.

Blumer, H. (1954): What's Wrong With Social Theory? In: American Sociological Review, 19(1), 3-10.

Bogner, A./Rosenthal, G. (2017): Biographien - Diskurse - Figurationen.

Methodologische Überlegungen aus einer sozialkonstruktivistischen und figurationssoziologischen Perspektive. In: Spies, T./Tuider, E. (Hrsg.):

Biographie und Diskurs. Methodisches Vorgehen und methodologische Verbindungen. Wiesbaden: Springer VS, 43-67.

Brand, L. (1988): Palestinians in Syria. The Politics of Integration. In: Middle East Journal, 42(4), 621-637.

Breckner, R. (2005): Migrationserfahrungen - Fremdheit - Biographie. Zum Umgang mit polarisierten Welten in Ost-West-Europa. Wiesbaden: VS. 
Castles, S. (2003): Towards a Sociology of Forced Migration and Social Transformation. In: Sociology, 37(1), 13-34.

Faist, T. (2000): Transstaatliche Räume. Wirtschaft, Politik und Kultur in und zwischen Deutschland und der Türkei. Bielefeld: transcript.

Fischer, W./Kohli, M. (1987): Biographieforschung. In: Voges, W. (Hrsg.):

Methoden der Biographie- und Lebenslaufforschung. Opladen:

Leske+Budrich, 25-49.

Fischer-Rosenthal, W. (1995): Schweigen, Rechtfertigen, Umschreiben.

Biographische Arbeit im Umgang mit deutschen Vergangenheiten. In: Fischer-

Rosenthal, W./Alheit, P. (Hrsg.): Biographien in Deutschland. Soziologische

Rekonstruktionen gelebter Gesellschaftsgeschichte. Wiesbaden: VS, 43-86.

Glick Schiller, N./Basch, L./Blanc-Szanton, C. (1992): Transnationalism. A New

Analytic Framework for Understanding Migration. In: Annals of the New

York Academy of Sciences, 645(1), 1-24.

Heller, C. (1995): Die Außenpolitik der Demokratischen Volksrepublik Algerien

1979 bis 1992. Marburg: Tectum Verlag.

Hess, S./Kasparek, B./Kron, S./Rodatz, M./Schwertl, M./Sontowski, S. (2017):

Der lange Sommer der Migration. Krise, Rekonstitution und ungewisse

Zukunft des europäischen Grenzregimes. In: Hess, S./Kasparek, B./Kron,

S./Rodatz, M./Schwertl, M./Sontowski, S. (Hrsg.): Der lange Sommer der

Migration. Grenzregime III. Berlin/Hamburg: Assoziation A, 6-24.

Hinnebusch, R. (2001): Syria. Revolution from Above. London: Routledge.

Hinnebusch, R. (2012): Syria: From 'Authoritarian Upgrading' to Revolution? In: International Affairs, 88(1), 95-113.

Hinrichsen, H./Rosenthal, G./Worm, A. (2013): Biographische

Fallrekonstruktionen: Zur Rekonstruktion der Verflechtung ,individueller“

Erfahrung, biographischer Verläufe, Selbstpräsentationen und „,kollektiver“

Diskurse. PalästinenserInnen als RepräsentantInnen ihrer Wir-Bilder. In:

Sozialer Sinn, 14(2), 157-184.

Hoffmann-Riem, C. (1980), Die Sozialforschung einer interpretativen Soziologie.

Der Datengewinn. In: Kölner Zeitschrift für Soziologie und Sozialpsychologie, 32, 339-372.

Inowlocki, L. (2002): Traditionalität als reflexiver Prozeß. Großmütter, Mütter und

Töchter in jüdischen Displaced-Persons-Familien. Eine biographieanalytische und wissenssoziologische Untersuchung. Habilitationsschrift, Otto-von-

Guericke-Universität Magdeburg. 
Kasparek, B. (2017): Europas Grenzen. Flucht, Asyl und Migration. Eine kritische Einführung. Berlin: Bertz+Fischer.

Kleist, J. O. (2015): Über Flucht forschen. Herausforderungen der

Flüchtlingsforschung. In: Peripherie, 35(138/139), 150-169.

Lefèvre, R. (2013): Ashes of Hama. The Muslim Brotherhood in Syria. New York: Oxford University Press.

Lutz, H. (1991): Welten verbinden. Türkische Sozialarbeiterinnen in den Niederlanden und der Bundesrepublik Deutschland. Frankfurt a. M.: IKO Verlag für Interkulturelle Kommunikation.

Perthes, V. (1990): Staat und Gesellschaft in Syrien. 1970-1989. Hamburg: Deutsches Orient Institut.

Pries, L. (1996): Transnationale Soziale Räume: Theoretisch-empirische Skizze am Beispiel der Arbeitswanderungen Mexico-USA. In: Zeitschrift für Soziologie, 6(25), 456-472.

Rosenthal, G. (1995): Erlebte und erzählte Lebensgeschichte. Gestalt und Struktur biographischer Selbstbeschreibungen. Frankfurt a. M./New York: Campus.

Rosenthal, G. (Hrsg.) (1997): Der Holocaust im Leben von drei Generationen. Familien von Überlebenden der Shoah und von Nazi-Tätern. Gießen: Psychosozial.

Rosenthal, G. (2004): ,Ethnisierung der Biographie‘ und Traumatisierung. In: Ottersbach, M./Yildiz, E. (Hrsg.): Migration in der metropolitanen Gesellschaft. Zwischen Ethnisierung und globaler Neuorientierung. Münster: Lit-Verlag, 217-227.

Rosenthal, G. (2015): Interpretative Sozialforschung. Eine Einführung. 5. Auflage. Weinheim/Basel: Beltz Juventa.

Rosenthal, G./Bahl, E./Worm, A. (2016): Illegalisierte Migrationsverläufe aus biografietheoretischer und figurationssoziologischer Perspektive. Die Landgrenze zwischen Spanien und Marokko. In: Forum Qualitative Sozialforschung, 17(3), Art. 10. Online: https://www.ssoar.info/ssoar/handle/ document $/ 57123<05.05 .2020>$.

Sauer, L./Diabaté, S./Gabel, S./Halfar, Y./Kraus, E./Wenzel, L. (2018): Doing transnational family im Kontext von Flucht und Krisenmigration. Stand der Forschung. BiB Working Paper 3. Online: https://www.bib.bund.de/Publikation/2018/pdf/Doing-transnational-familyim-Kontext-von-Flucht-und-Krisenmigration-Stand-der-Forschung.html $<28.04 .2020>$. 
Schütz, A. (1971[1953]): Wissenschaftliche Interpretation und Alltagsverständnis menschlichen Handelns. In: Gesammelte Aufsätze I. Das Problem der sozialen Wirklichkeit. Den Haag: Nijhoff, 3-54.

Schütze, F. (1977): Die Technik des narrativen Interviews in Interaktionsfeldstudien. Dargestellt an einem Projekt zur Erforschung kommunaler Machtstrukturen. In: Arbeitsberichte und Forschungsmaterialien Nr. 1 der Universität Bielefeld.

Shami, S. (1996): Transnationalism and Refugee Studies. Rethinking Forced Migration and Identity in the Middle East. In: Journal of Refugee Studies, 9(1), $3-26$.

Siouti, I. (2013): Transnationale Biographien. Eine biographieanalytische Studie über Transmigrationsprozesse bei der Nachfolgegeneration griechischer Arbeitsmigranten. Bielefeld: transcript.

Stepputat, F./Sørensen, N. (2014): Sociology and Forced Migration. In: FiddianQasmiyeh, E./Loescher, G./Long, K./Sigona, N. (Hrsg.): The Oxford Handbook of Refugee and Forced Migration Studies. Oxford: Oxford University Press, 86-98.

Thomas, W. I./Znaniecki, F. (1958[1918-1920]): The Polish Peasant in Europe and America. New York: Dover.

Walters, W. (2004): The Frontiers of the European Union. A Geostrategic Perspective. In: Geopolitics, 9(3), 674-698.

Wimmer, A./Glick Schiller, N. (2002): Methodological Nationalism and Beyond. Nation-State Building, Migration and Social Sciences. In: Global Networks, 2(4), 301-334.

Worm, A. (2019): Fluchtmigration aus Syrien. Eine biographietheoretische und figurationssoziologische Studie. Göttinger Beiträge zur soziologischen Biographieforschung, Bd. 1. Göttingen: Universitätsverlag Göttingen. Online: https://doi.org/10.17875/gup2019-1228<09.04.2020>. 